\title{
Non-Anti-Neutrophil Anti-Cytoplasmic Antibodies Associated Methimazole-Induced Nephritis: Case and Review of Literature
}

\author{
Hengyi Guo ${ }^{\mathrm{a}}$, David Levitt ${ }^{\mathrm{b}}$, Kashif M. Munir ${ }^{\mathrm{b}}$, Cynthia B. Drachenberg ${ }^{\mathrm{c}}$, \\ Rana Malek ${ }^{b}$, Elizabeth M. Lamos ${ }^{\text {b, d }}$
}

\begin{abstract}
Current anti-thyroid therapy is rarely associated with adverse effects such as vasculitis or nephritis. Historically, vasculitis associated with anti-thyroid therapy is associated with anti-neutrophil anti-cytoplasmic antibodies (ANCA). Here, we report a rare case of ANCA-negative interstitial nephritis secondary to methimazole, resulting in end organ renal failure. A 27-year-old male with a history of Graves' hyperthyroidism on methimazole anti-thyroid therapy presented to the hospital with shortness of breath and chest pain, complicated by a pericardial effusion and acute kidney injury (AKI) requiring emergent hemodialysis. The diagnosis of drug-induced ANCA-negative vasculitis was based on the temporal relationship between clinically evident vasculitis and treatment with methimazole, after excluding other causes of vasculitis. The lack of ANCA-associated positivity should not deter clinicians from considering stopping the offending agent and pursuing more definitive therapy (radioactive iodine or surgery as appropriate).
\end{abstract}

Keywords: Methimazole; Interstitial nephritis; Renal failure; ANCA-negative vasculitis

\section{Introduction}

Methimazole (MMI), carbimazole, and propylthiouracil (PTU) are anti-thyroid medications belonging to the class of thion-

Manuscript submitted April 12, 2018, accepted April 19, 2018

anniversity of Maryland School of Medicine, $655 \mathrm{~W}$ Baltimore S, Baltimore, MD 21201, USA

bivision of Endocrinology, Diabetes and Nutrition, University of Maryland School of Medicine, 827 Linden Ave, 2nd Floor, Baltimore, MD 21201, USA cUniversity of Maryland School of Medicine, 22 South Greene St., Baltimore, MD 21201, USA

${ }^{\mathrm{d}}$ Corresponding Author: Elizabeth M. Lamos, Division of Endocrinology, Diabetes and Nutrition, University of Maryland School of Medicine, 827 Linden Ave, 2nd Floor, Baltimore, MD 21201, USA.

Email: elamos@som.umaryland.edu

doi: https://doi.org/10.14740/jem505w amides. MMI, the active metabolite of carbimazole, is the first-line medical treatment for hyperthyroidism in the United States. It is generally considered safe. The main adverse effect is a rash, but infrequent cases of agranulocytosis and hepatotoxicity have been reported [1]. Current anti-thyroid medications are rarely associated with vasculitis or interstitial nephritis, but reported literature suggests that PTU is more frequently linked to these adverse reactions than MMI [2].

Historically, the vasculitis associated with PTU or MMI is associated with anti-neutrophil anti-cytoplasmic antibodies (ANCA), which are sensitive and specific markers for systemic vasculitis and pauci-immune necrotizing/crescentic glomerulonephritis [3]. ANCA can be helpful in determining etiologies of small vessel vasculitis. History and examination can often distinguish drug-induced vasculitis versus other etiologies such as granulomatosis with polyangiitis, microscopic polyangiitis, and Churg-Strauss syndrome. ANCA-positive drug-induced vasculitis is generally diagnosed within days to weeks of drug exposure and associated with skin manifestations [4].

In the setting of a small-vessel vasculitis in which ANCA is negative, the differential diagnosis is larger. Additional features such as cryoglobulin and immunoglobulin deposits can be helpful. If the history and physical do not elucidate the etiology, drug-induced immune complex vasculitis can be suspected under the right conditions.

Here we report a rare case of ANCA-negative interstitial nephritis secondary to MMI, resulting in end organ renal failure. This case highlights the challenging diagnosis of drug-induced vasculitis in the setting of negative immune markers. The limited literature of ANCA-negative vasculitis associated with any anti-thyroid therapy will be reviewed. Finally, the importance of recognizing drug-induced vasculitis in the absence of ANCA and ways to manage it will be discussed.

\section{Case Report}

A 27-year-old African American male with a history of Graves' hyperthyroidism presented to the hospital with shortness of breath and chest pain, complicated by a pericardial effusion 
Table 1. Significant Lab Values on Admission Compared to Baseline

\begin{tabular}{lll}
\hline Lab and normal range & Baseline & On hospital admission \\
\hline Creatinine $(0.66-1.25 \mathrm{mg} / \mathrm{dL})$ & 0.84 & $21.9(\mathrm{H})$ \\
BUN $(6-20 \mathrm{mg} / \mathrm{dL})$ & 10 & $28(\mathrm{H})$ \\
Urine analysis & & $3+(\mathrm{H})$ \\
$\quad$ Protein $($ trace $)(10-20 \mathrm{mg} / \mathrm{dL})$ & Trace & $26-50(\mathrm{H})$ \\
$\quad$ WBC $(0-5 / \mathrm{hpf})$ & $0-5$ & $3-5(\mathrm{H})$ \\
\hline RBC $(0-2 / \mathrm{hpf})$ & $0-2$ & \\
\hline
\end{tabular}

$\mathrm{H}$ : high.

and acute kidney injury (AKI) requiring emergent hemodialysis. Six months earlier, he was diagnosed with Graves' hyperthyroidism and was started on MMI $10 \mathrm{mg} /$ day, but he reported intermittent non-adherence. At the time of diagnosis and administration of MMI, the patient was otherwise healthy with no baseline renal dysfunction.

Upon admission to the hospital, fever $\left(38.2^{\circ} \mathrm{C}\right)$, prominent proptosis, and goiter were present. No lower extremity edema, dry eyes, rash, joint pain, or myalgia were reported or noted on examination. Significant lab findings are presented in Tables 1 and 2. The following findings are highlighted: thyroid-stimulating hormone (TSH) was decreased and free thyroxine was elevated consistent with hyperthyroidism. Renal laboratory results demonstrated severe renal insufficiency with elevated urea and creatinine. C-reactive protein (CRP) and erythrocyte sedimentation rate (ESR) were also elevated.

Table 2. Significant Lab Values on Admission With No Baseline Comparison

\begin{tabular}{|c|c|}
\hline Lab and normal range & $\begin{array}{l}\text { On hospital } \\
\text { admission }\end{array}$ \\
\hline SSA AB $(0-0.9 \mathrm{ZZ})$ & $>8.0(\mathrm{H})$ \\
\hline SSB AB $(0-0.9 \mathrm{ZZ})$ & $>8.0(\mathrm{H})$ \\
\hline TSH (0.47 - $4.68 \mathrm{mIU} / \mathrm{L})$ & $0.005(\mathrm{~L})$ \\
\hline Free thyroxine $(0.7-1.9 \mathrm{ng} / \mathrm{dL})$ & $2.41(\mathrm{H})$ \\
\hline Total T4 (5.1 - $13.8 \mu \mathrm{g} / \mathrm{dL})$ & 7.5 \\
\hline Urea $(9-20 \mathrm{mg} / \mathrm{dL})$ & $211(\mathrm{H})$ \\
\hline C-reactive protein $(0-10 \mathrm{mg} / \mathrm{dL})$ & $11(\mathrm{H})$ \\
\hline CRP high sensitivity $(<3.00 \mathrm{mg} / \mathrm{L})$ & $>15.00(\mathrm{H})$ \\
\hline Erythrocyte sedimentation rate $(0-15 \mathrm{~mm} / \mathrm{h})$ & $119(\mathrm{H})$ \\
\hline LDH (313 - 618 units/L) & $778(\mathrm{H})$ \\
\hline Amylase (48 - 133 units/L) & $269(\mathrm{H})$ \\
\hline Lipase (23 - 300 units/L) & $402(\mathrm{H})$ \\
\hline $\mathrm{C} 3(90-165 \mathrm{mg} / \mathrm{dL})$ & $85(\mathrm{~L})$ \\
\hline $\mathrm{C} 4(10-40 \mathrm{mg} / \mathrm{dL})$ & 28 \\
\hline Total complement CH50 (42 - $62 \mathrm{U} / \mathrm{mL})$ & $66(\mathrm{H})$ \\
\hline Histopathological IgG4 plasma cells ( $>10 / \mathrm{hpf}$ ) & $19(\mathrm{H})$ \\
\hline Gamma globulin fraction $(0.4-1.6 \mathrm{~g} / \mathrm{dL})$ & $2.0(\mathrm{H})$ \\
\hline
\end{tabular}

$\mathrm{H}$ : high.
Liver function tests including alkaline phosphatase and alanine transferase were normal. Urinalysis revealed $3+$ protein, elevated WBC, and presence of RBC. Subsequently, it was determined that both ANA (1:640 titer) with speckled pattern and SSA/SSB were positive. However, a lip biopsy was not consistent with Sjogren's disease. Further negative findings included P-ANCA, C-ANCA, dsDNA Ab, RPR, anti-GBM, anti-Smith, B2 glycoprotein, cryoglobulin, RNP, HIV, HBV, $\mathrm{HCV}$, anti-cardiolipin, SPEP, and UPEP. Polyclonal hypergammaglobulinemia was noted. Measurement of complement showed a low $\mathrm{C} 3$ level, normal $\mathrm{C} 4$, but total increased complement (CH50). Serological tests showed no hepatitis $\mathrm{C}$ infection.

In addition, an initial chest radiograph showed moderate bilateral pleural effusions, while an echocardiogram noted a large $(1.8 \times 1.8 \mathrm{~cm})$ circumferential pericardial effusion with possible fibrinous stranding, features consistent with cardiac tamponade and possible pericarditis.

On renal biopsy (Fig. 1), 13 of the 16 glomeruli in the renal cortex showed global sclerosis, and two of the remaining glomeruli showed extra capillary proliferations consisting of an epithelial and a fibroepithelial crescent. The glomerular process was pauci-immune. The interstitium contained extensive, confluent mononuclear inflammatory infiltrates composed predominantly of lymphocytes and macrophages with a minor, but significant component of polyclonal plasma cells and scattered eosinophils. Multifocal tubular inflammation (tubulitis) was noted with prominent tubular atrophy and associated reactive epithelial changes. Granulomas and features diagnostic of $\mathrm{IgG} 4$ nephropathy were not found. The arteries were normal.

The acute and chronic tubulointerstitial nephritis and pauci-immune crescentic glomerulonephritis were likely related to MMI use. This impression was supported by the temporal relation of symptoms to the commencement and duration of treatment with MMI. Thus, MMI was discontinued and hemodialysis was initiated. Subsequently, severe hyperthyroidism ensued and was treated with IV dexamethasone and betablockade. Total thyroidectomy was performed during the same admission to avoid further exposure to MMI. The patient was discharged on weight-based thyroid hormone replacement therapy (levothyroxine $125 \mathrm{mcg}$ daily), and a tapered dose of prednisone. The pleural effusion and pericardial effusion completely resolved rapidly with steroid treatment. The patient remained hemodialysis dependent due to irreversible kidney 


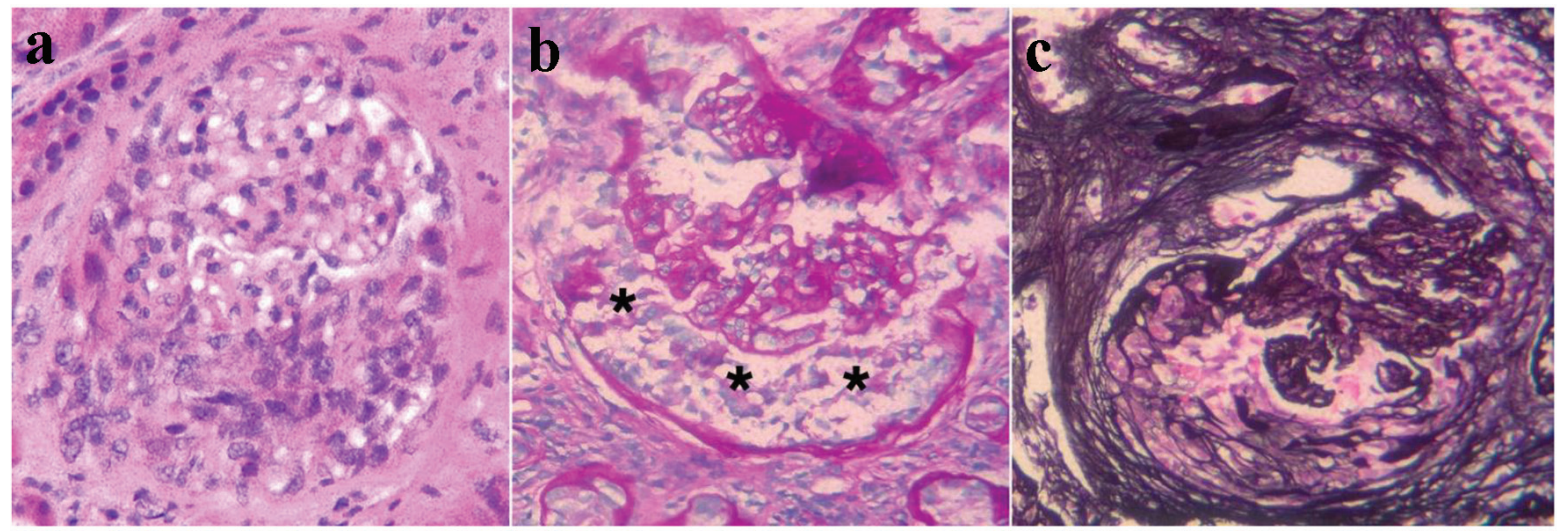

Figure 1. Representative images from the kidney biopsy demonstrate glomerular crescents. (a) An epithelial crescent is seen in the lower half of the glomerular area (H\&E stain). (b) The PAS stain highlights the glomerular tufts and the Bowman's capsule. The urinary space between these structures contains an epithelial crescent (asterisks). (c) The Jones silver stain highlights an organizing crescent (fibro-epithelial crescent) in the left and lower glomerular areas. The glomerular tufts appear compressed.

damage.

\section{Discussion}

ANCA-positive vasculitis secondary to anti-thyroid drugs has been reviewed and described previously [5]. Of the antithyroid drugs, MMI is less likely to be associated with this adverse reaction compared to carbimazole $[6,7]$. There is a paucity of literature describing ANCA-negative anti-thyroid drug-induced vasculitis. Kanat et al reported one case of MMIinduced leukocytoclastic vasculitis [8]. Additionally, Day et al described one case of ANCA-negative interstitial nephritis associated with carbimazole [9]. Table 3 compares our present case with two other similar cases: Kanat et al and Day et al.

In comparison, all three cases were men, aged 56, 72, and
27 who were diagnosed with hyperthyroidism. The etiology of the hyperthyroidism was not reported in the historical cases; however, our patient's disease was secondary to Grave's autoimmune disease. They all developed a vasculitic reaction to either MMI or its pro-drug form carbimazole, but after various durations of drug use. The historical cases were associated with much shorter duration of therapy compared to our case. Our case is most similar to Day et al's in that both individuals suffered interstitial nephritis confirmed by renal biopsies with elevated creatinine (21.9 and $6.23 \mathrm{mg} / \mathrm{dL}$, respectively), subsequently developing irreversible kidney damage and requiring permanent hemodialysis. In both cases, the offending drug was stopped, and steroids were administered. Day et al and Kanat et al's cases presented with rash while our case did not. The individual described by Kanat et al demonstrated vasculitic skin manifestations which resolved with the cessation of MMI and

Table 3. A Comparison of Findings Among the Three Papers

\begin{tabular}{llll}
\hline Case number & $\mathbf{1}[\mathbf{8}]$ & $\mathbf{2}[\mathbf{9}]$ & Present case \\
\hline Presence of rash & Yes & Yes & No \\
ANCA positivity & No & No & No \\
ANA positivity & No & N/A & Yes \\
Anti-dsDNA positivity & No & N/A & No \\
Anti-glomerular basement membrane positivity & N/A & No & No \\
Serum cryoglobulin & N/A & Yes & No \\
Hepatitis C & N/A & No & No \\
C3 levels & N/A & Low & Low \\
C4 levels & N/A & Low & WNL \\
Other findings & CRP 23.7 (H) & CRP 114 mg/L (H) & CRP 14 mg/L (H) \\
& ESR WNL & ESR 25 mm/h (H) & ESR 119 mm/h (H) \\
& & Creatinine 6.32 mg/dL (H) & Creatinine 21.9 mg/dL (H) \\
& & & SSA/SSB negative \\
\end{tabular}

ANA: anti-neutrophil; ANCA: anti-neutrophil anti-cytoplasmic antibodies; C: complement; CRP: C-reactive protein; ESR: erythrocyte sedimentation rate; N/A: presence or absence of finding not specified. 
Table 4. Summary of Causes and Outcomes of Vasculitis or Interstitial Nephritis Induced by Methimazole or Its Pro-Drug Form Carbimazole in Reported Cases

\begin{tabular}{|c|c|c|c|}
\hline Case number & 1 & 2 & 3 \\
\hline Author & Kanat et al [8] & Day et al [9] & Present case \\
\hline Patient age (years)/sex & $56 / \mathrm{M}$ & $72 / \mathrm{M}$ & $27 / \mathrm{M}$ \\
\hline Medication dosage/duration & MMI 20 mg daily/1 week & Carbimazole $20 \mathrm{mg}$ daily/2 weeks & MMI 10 mg daily/6 months \\
\hline Biopsy findings & $\begin{array}{l}\text { 1) Cutaneous } \\
\text { leukocytoclastic vasculitis }\end{array}$ & $\begin{array}{l}\text { 1) Cutaneous leukocytoclastic vasculitis } \\
\text { 2) Interstitial nephritis w/eosinophils }\end{array}$ & $\begin{array}{l}\text { 1) Chronic tubulointerstitial } \\
\text { nephritis w/crescentic changes }\end{array}$ \\
\hline Thyroidectomy & No & No & Yes \\
\hline Outcome & $\begin{array}{l}\text { 1) Cutaneous } \\
\text { rashes resolved }\end{array}$ & $\begin{array}{l}\text { 1) Cutaneous rash improved } \\
\text { 2) HD dependent }\end{array}$ & $\begin{array}{l}\text { 1) Pleural effusions resolved } \\
\text { 2) HD dependent }\end{array}$ \\
\hline
\end{tabular}

HD: hemodialysis; MMI: methimazole.

treatment with steroids.

The diagnosis of drug-induced ANCA-negative vasculitis is based on the temporal relationship between clinically evident vasculitis and treatment with the offending drugs, after excluding other causes of vasculitis [10]. The cause of the interstitial nephritis in our patient was determined to be secondary to MMI, especially having eliminated other potential causes. Immune testing and biopsy as well as history were not consistent with other possible ANCA-negative vasculitis etiologies. For example, chronic interstitial nephritis is one of the most common renal manifestations of Sjogren's syndrome [11]. Thus, Sjogren's syndrome was considered a possible cause of his renal failure given his history of autoimmunity and serological profile (SSA- and SSB-positive); however, it was deemed unlikely after a negative lip biopsy. Another potential cause of acute interstitial nephritis that was considered was mixed cryoglobulinemia. SSA/SSB and positive cryoglobulinemia were both characteristics described in Day et al's carbimazole-induced vasculitis. In our patient, however, cryoglobulin quantity was negative and there was no other clear supporting evidence for cryoglobulinemia such as skin lesions including palpable purpura, ulcers, and areas of necrosis that occur in $69-89 \%$ of mixed cryoglobulinemic disease [12]. There was no evidence of cryoglobulin-associated glomerulonephritis. Furthermore, the most common cause of cryoglobulinemia is hepatitis C infection [13] and serology was negative in our patient. Also, the possibility of hypergammaglobulinemia and hypocomplementemia were considered as other potential causes of interstitial nephritis. IgG4 disease has a "storiform" pattern (cartwheel pattern) of fibrosis and was initially described in 2003 and reported to cause interstitial nephritis in 2004 [14, 15]. However, in our patient, while his renal biopsy had some IgG4-positive plasma cells, there was no storiform fibrosis. Serum IgG4 was not measured. Hypocomplementemic tubulointerstial nephritis has only been described in several case reports and is characterized by a large amount of tubulointerstitial deposits and lymphoid or plasma cell infiltration on kidney biopsy and systemic hypocomplementemia [16]. Although plasma complement C3 levels were low in our patient, C4 was normal, and total complement levels (CH50) were elevated.

Other evidence supports a drug-induced interstitial nephritis diagnosis. For example, the dense tubular casts in our patients is a finding that is consistent with drug-induced interstitial nephritis. In addition, our patient's pericardial effusion may also be secondary to drug-induced vasculitis. There have been five cases of pleural effusion induced by anti-thyroid drugs, two with PTU $[17,18]$ and three with carbimazole [1921], that have been reported in the literature. It is unknown whether there were eosinophils in his pleural effusions, which if present, would be more evidence for a potential drug cause.

In addition, one of the fundamental challenges of this case and other similar cases is proving ANCA negativity, which is often difficult to do. One of the reasons that ANCA-negative vasculitis remains challenging to determine is the length of time it can take for ANCA to develop. From symptom onset, it may take up to 3 months for ANCA to be detected [22] and may not ever become evident. Thus, this diagnosis becomes one of exclusions.

In our patient, the pericardial and pleural effusions did improve rapidly, but renal function did not recover with cessation of MMI and high dose glucocorticoids (Table 4). The persistent renal dysfunction may be partially explained by the phenomenon that ANCA-negative patients also have a higher prevalence of nephritic syndrome and poorer renal survival than ANCA-positive patients [23]. However, the pathogenesis of ANCA-negative crescentic glomerulonephritis is still unclear.

This case illustrates that although rare, drug-induced microscopic vasculitis with crescentic glomerulonephritis associated with anti-thyroid medications, MMI in this case, can be difficult to diagnose. Although there is no current recommendation [24] to monitor renal function or blood counts in treatment of hyperthyroidism, there should be a low threshold to consider drug-induced dysfunction should the appropriate clinical scenario present itself. The lack of ANCA-associated positivity should not deter clinicians from considering stopping the offending agent and pursuing more definitive therapy (radioactive iodine or surgery as appropriate). Surrogate infor- 
mation such as eosinophils in body fluids, biopsy of end organs with dysfunction (i.e. skin or kidney) may be helpful. Drug reactions to anti-thyroid medications are more likely early in therapy, but our case demonstrated prolonged exposure to antithyroid therapy.

In summary, we report the case of a 27-year-old male who developed ANCA-negative crescentic glomerulonephritis and acute and chronic interstitial nephritis after a 6-month course of treatment with MMI. Because of the temporal relationship with the commencement of MMI, the absence of ANCA, and other causes of interstitial nephritis ruled out, we propose these changes were caused by a drug reaction to MMI which developed over time. Although this response has not been reported previously, this case draws attention to this potential side effect of MMI given its widespread use. It is important for physicians to be aware that the interval between the first administration of a drug and the development of vasculitis is extremely variable between drugs as well as the individuals receiving these agents [4]. Awareness of drug-induced vasculitis is important to make a prompt diagnosis, start steroid treatment, and thus achieve the most optimal possible outcome.

\section{References}

1. Bartalena L, Bogazzi F, Martino E. Adverse effects of thyroid hormone preparations and antithyroid drugs. Drug Saf. 1996;15(1):53-63.

2. Noh JY, Yasuda S, Sato S, Matsumoto M, Kunii Y, Noguchi Y, Mukasa K, et al. Clinical characteristics of myeloperoxidase antineutrophil cytoplasmic antibody-associated vasculitis caused by antithyroid drugs. J Clin Endocrinol Metab. 2009;94(8):2806-2811.

3. Savage CO, Pusey CD, Bowman C, Rees AJ, Lockwood CM. Antiglomerular basement membrane antibody mediated disease in the British Isles 1980-4. Br Med J (Clin Res Ed). 1986;292(6516):301-304.

4. ten Holder SM, Joy MS, Falk RJ. Cutaneous and systemic manifestations of drug-induced vasculitis. Ann Pharmacother. 2002;36(1):130-147.

5. Morita S, Ueda Y, Eguchi K. Anti-thyroid drug-induced ANCA-associated vasculitis: a case report and review of the literature. Endocr J. 2000;47(4):467-470.

6. Yazbeck R, Francoual J, Benoit P, Tauveron I, Tchen C, Thieblot P. [Carbimazole and leukocytoclastic vasculitis: apropos of a case]. Rev Med Interne. 1999;20(4):350352 .

7. Miller RM, Savige J, Nassis L, Cominos BI. Antineutrophil cytoplasmic antibody (ANCA)-positive cutaneous leucocytoclastic vasculitis associated with antithyroid therapy in Graves' disease. Australas J Dermatol. 1998;39(2):96-99.

8. Kanat M, Goksugur N, Parlak A. A case of methimazole induced leukocytoclastic vasculitis. Turkish J Endocrinol Metab. 2005;4:125-127.

9. Day C, Bridger J, Rylance P, Jackson M, Nicholas J, Odum J. Leukocytoclastic vasculitis and interstitial ne- phritis with carbimazole treatment. Nephrol Dial Transplant. 2003;18(2):429-431.

10. Radic M, Martinovic Kaliterna D, Radic J. Drug-induced vasculitis: a clinical and pathological review. Neth J Med. 2012;70(1):12-17.

11. Kidder D, Rutherford E, Kipgen D, Fleming S, Geddes C, Stewart GA. Kidney biopsy findings in primary Sjogren syndrome. Nephrol Dial Transplant. 2015;30(8):13631369.

12. Muchtar E, Magen H, Gertz MA. How I treat cryoglobulinemia. Blood. 2017;129(3):289-298.

13. Lauletta G, Russi S, Conteduca V, Sansonno L. Hepatitis $\mathrm{C}$ virus infection and mixed cryoglobulinemia. Clin Dev Immunol. 2012;2012:502156.

14. Takeda S, Haratake J, Kasai T, Takaeda C, Takazakura E. IgG4-associated idiopathic tubulointerstitial nephritis complicating autoimmune pancreatitis. Nephrol Dial Transplant. 2004;19(2):474-476.

15. Saeki T, Saito A, Yamazaki H, Emura I, Imai N, Ueno $\mathrm{M}$, Nishi S, et al. Tubulointerstitial nephritis associated with IgG4-related systemic disease. Clin Exp Nephrol. 2007;11(2):168-173.

16. Kambham N, Markowitz GS, Tanji N, Mansukhani MM, Orazi A, D'Agati VD. Idiopathic hypocomplementemic interstitial nephritis with extensive tubulointerstitial deposits. Am J Kidney Dis. 2001;37(2):388-399.

17. Middleton KL, Santella R, Couser JI, Jr. Eosinophilic pleuritis due to propylthiouracil. Chest. 1993;103(3):955956.

18. Sen N, Ermis H, Karatasli M, Habesoglu MA, Eyuboglu FO. Propylthiouracil-associated eosinophilic pleural effusion: a case report. Respiration. 2007;74(6):703-705.

19. Das G, Stanaway SE, Brohan L. Carbimazole induced pleural effusion: a case report. Case Rep Endocrinol. 2012;2012:941241.

20. Haq I, Sosin MD, Wharton S, Gupta A. Carbimazoleinduced lupus. BMJ Case Rep. 2013;2013.

21. Cardona Attard CD, Gruppetta M, Vassallo J, Vella S. Carbimazole-induced exudative pleural effusions. BMJ Case Rep. 2016;2016.

22. Bonaci-Nikolic B, Nikolic MM, Andrejevic S, Zoric S, Bukilica M. Antineutrophil cytoplasmic antibody (ANCA)-associated autoimmune diseases induced by antithyroid drugs: comparison with idiopathic ANCA vasculitides. Arthritis Res Ther. 2005;7(5):R1072-1081.

23. Morikawa T, Yoshida A, Kobayashi S, Shibata M, Hamada M, Kishida M, Kitabayashi C, et al. AP-VAS 2012 case report: a case of ANCA-negative pauci-immune crescentic glomerulonephritis associated with IL-6-producing adenosquamous cell carcinoma of the lung. CEN Case Rep. 2013;2(2):158-164.

24. Bahn RS, Burch HB, Cooper DS, Garber JR, Greenlee $\mathrm{MC}$, Klein I, Laurberg P, et al. Hyperthyroidism and other causes of thyrotoxicosis: management guidelines of the American Thyroid Association and American Association of Clinical Endocrinologists. Endocr Pract. 2011;17(3):456-520. 